\title{
Evolução do Carcinoma Colorretal, Comparando Doentes com Idades Acima e Abaixo de 40 Anos, Quanto à Diferenciação Tumoral e ao Estádio do Tumor
}

\author{
Colorectal Cancer Evolution, Comparing Patients Yourger and Older than \\ 40 Years Old, According to Tumoral Differentiation and Tumor Stage
}

\author{
LUIS ROBERTO MANZIONE NADAL ${ }^{1}$; CAROLINE TERUMI ADACHI'; MARCUS AURELIUS ARAUJO NUNES ${ }^{1}$;

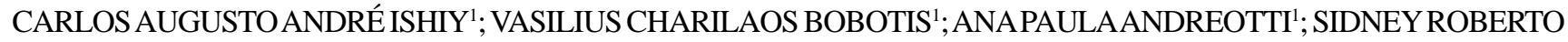

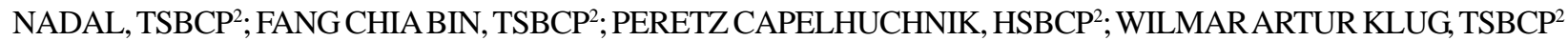 \\ ${ }^{1}$ Membro da Liga de Coloproctologia, Acadêmico da Faculdade de Ciências Médicas da Santa Casa de São Paulo; \\ ${ }^{2}$ Disciplina de Coloproctologia do Departamento de Cirurgia da Faculdade de Ciências Médicas da Santa Casa de \\ São Paulo.
}

\begin{abstract}
NADAL LRM; ADACHI CT; NUNES MAA; ISHIY CAA; BOBOTIS VC; ANDREOTTI AP; NADAL SR; CAPELHUCHNIK P; KLUG WA. Evolução do Carcinoma Colorretal, Comparando Doentes com Idades Acima e Abaixo de 40 Anos, Quanto à Diferenciação Tumoral e ao Estádio do Tumor. Rev bras Coloproct, 2009;29(3): 351-357.

RESUMO: Objetivo: A incidência elevada do carcinoma colorretal o torna problema de saúde pública no nosso país. Os poucos trabalhos na literatura, bem como as dúvidas relacionando a idade com a evolução da doença, estimularam-nos a realizar esse trabalho para conhecer as divergências quanto à diferenciação tumoral e o estádio na evolução dessa neoplasia, comparando doentes com idades acima e abaixo de 40 anos. Método: Comparar 205 doentes de adenocarcinoma colorretal com idades acima e abaixo de 40 anos quanto ao tempo de sintomas, história familiar, localização do tumor, estádio do tumor, diferenciação, morte operatória, local de metástases e mortalidade até 3 anos. Resultados: Eram 20 no grupo mais jovem e 185 entre os mais idosos. Não houve diferença em relação ao sexo, ao tempo de início de sintomas, à história familiar, ao local de tumor no cólon, ao estádio, ao aparecimento de recidivas, à mortalidade operatória e à sobrevivência até o terceiro ano pós-operatório. No grupo mais jovem os tumores foram mais indiferenciados e as metástases abdominais predominaram. No grupo mais velho houve maior incidência de metástases hepáticas e pulmonares. Conclusão: Os resultados obtidos nas condições de execução do presente estudo, em que comparamos doentes portadores de adenocarcinoma colorretal com idades acima e abaixo de 40 anos, permitiram concluir que os tumores foram mais indiferenciados entre os mais jovens embora a evolução pós-tratamento tenha sido semelhante.
\end{abstract}

Descritores: Adenocarcinoma, Neoplasias Colorretais, Estadiamento de Neoplasias, Diferenciação celular.

\section{INTRODUÇÃO}

O carcinoma colorretal (CCR) é das neoplasias mais frequentes na população adulta mundial, sendo que 600.000 casos são diagnosticados por ano. No Brasil, é o quinto tumor maligno mais frequente entre os homens e $o$ quarto entre as mulheres. ${ }^{4}$ Dentre as do trato gastrointestinal é a segunda em prevalência e mortalidade. ${ }^{1,2}$ Vem apresentando incidências progressivamente maiores segundo estudos realizados nos últimos 15 anos. ${ }^{3}$ Entretanto, a distribuição em relação a sexo e idade se mantém constante, acometendo homens na proporção de 1 para 0,8 mulheres na faixa etária entre 50 e 80 anos. ${ }^{3}$

O CCR predomina em indivíduos acima de 50 anos $^{5}$ e entre $2 \%$ e $10,6 \%$ dos diagnósticos são feitos em pacientes abaixo dos 40 anos, ${ }^{6}$ sendo $2,4 \%$ quando abaixo dos 30 anos, ${ }^{7}$ embora estudos japoneses mostrem porcentagens maiores, de $10 \%$ a $14 \% .^{8-12}$ 
Alguns estudos têm demonstrado que doentes mais jovens apresentam prognóstico pior, com sobrevivência menor comparativamente aos mais idosos, ${ }^{13-15}$ fato não observado por outros. ${ }^{16,17}$ Todavia, os fatores para esse pior prognóstico seriam o estádio mais avançado da doença ao diagnóstico ${ }^{18,19} \mathrm{e}$ a presença de tumores menos diferenciados. ${ }^{20}$

Quando se compararam os estádios da doença em indivíduos mais jovens e mais velhos que 40 anos, afirmaram que nos mais jovens a doença apresentouse em estádio mais avançado. ${ }^{8-11,15}$ Todavia, outros estudos não observaram essa diferença., ${ }^{2,21}$ Também é sabido que quanto mais indiferenciado pior o prognóstico, ${ }^{22}$ embora haja pouca evidência sobre esse aspecto. $^{23}$

A incidência elevada do CCR o torna problema de saúde pública no nosso país. Os poucos trabalhos na literatura, bem como as dúvidas relacionando a idade com a evolução da doença, estimulou-nos a realizar esse trabalho, para conhecer as divergências quanto à diferenciação tumoral e o estádio na evolução dessa neoplasia, comparando doentes com idades acima e abaixo de 40 anos.

\section{MÉTODO}

Fizemos estudo transversal e retrospectivo com análise de prontuários de 205 doentes submetidos a tratamento cirúrgico de CCR no período entre 2000 e 2005. Eram 113 homens (55\%) e 92 mulheres (45\%), com idades oscilando entre 22 e 89 anos, com maior prevalência entre as sexta e oitava décadas da vida.

Agrupamos os doentes em duas faixas etárias principais: Grupo 1, doentes com idade até 40 anos, e Grupo 2, doentes mais velhos que 40 anos. Comparamos o tempo de sintomas até o diagnóstico, a história familiar considerando doença em qualquer órgão ou parente das linhas horizontal e vertical, a localização do tumor no segmento colorretal. Para facilitar a avaliação quanto ao sítio do tumor, agrupamos os localizados no ceco, no cólon ascendente, no ângulo hepático e no cólon transverso sob o título de cólon direito; aqueles situados no ângulo esplênico e cólon descendente como cólon esquerdo; os do reto e os do sigmoide que ficaram em outro grupo, o estádio do tumor, utilizando a classificação TNM (UICC), o grau de diferenciação tumoral, comparando tumores bem e moderadamente diferenciados com aqueles mais indiferenciados, incluindo a presença do componente mucinoso e a presen- ça de células em anel de sinete, a mortalidade operatória computando as mortes até o $30^{\circ}$ dia de pós-operatório, o local de aparecimento de metástases, o aparecimento de recidivas, a sobrevivência em 3 anos

Os resultados foram avaliados com os métodos estatísticos adequados, com índice de rejeição da hipótese de nulidade de 5\%.

\section{RESULTADOS}

Eram 20 doentes $(9,75 \%)$ com idade inferior a 40 anos (grupo 1) e 185 mais velhos $(90,25 \%$ ) (grupo 2). No primeiro grupo, 13 eram homens $(65 \%)$ e sete mulheres $(35 \%)$ com média etária de 33,6 anos e no grupo 2 eram 186 homens (53\%) e 83 mulheres (47\%), sem diferença estatística $(p=0,49)$. (Gráfico 1)

Dentre as queixas que levaram os doentes a procurar atendimento médico, a enterorragia e a dor abdominal foram as mais comuns. O tempo de início dos sintomas variou entre 3 dias e 4 anos. Observamos que a maioria dos doentes mais idosos procurou atendimento em até três meses do início dos sintomas enquanto que no grupo mais jovem a distribuição foi mais equilibrada. (Tabela 1). A análise estatística pelo méto-

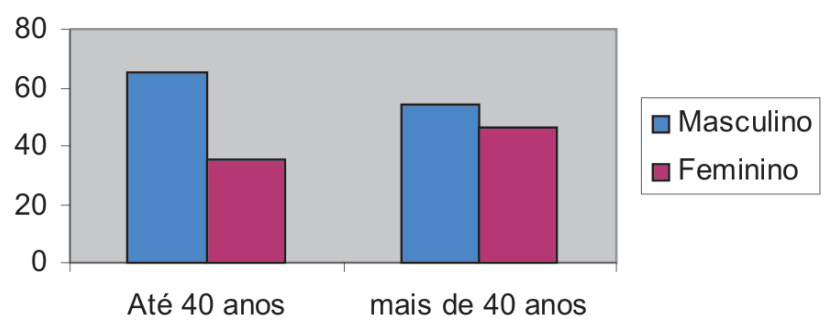

Gráfico 1 - Distribuição percentual de 205 pacientes tratados de câncer colorretal conforme sexo e idade.

Tabela 1 - Distribuição de 205 doentes de carcinoma colorretal conforme o grupo etário e o tempo de início de sintomas. 2006.

\begin{tabular}{lccc}
\hline Tempo & Grupo 1 & Grupo 2 & $\boldsymbol{p}$ \\
\hline Até $3 \mathrm{~m}$ & 4 & 83 & 0,07 \\
4 a 6 m & 3 & 26 & 0,92 \\
7 a 12 & 4 & 30 & 0,79 \\
> 1 ano & 5 & 21 & 0,11 \\
s/ resp & 4 & 21 & - \\
Total & 20 & 181 & - \\
\hline
\end{tabular}


do da comparação das porcentagens não mostrou diferença, $(p=0,13)$ embora haja tendência a que no grupo 2 a procura pelo médico seja mais precoce. $(p=$ $0,07)$

A história familiar de câncer foi identificada em $50 \%$ dos doentes do grupo 1 e em $31,5 \%$ no grupo 2. Entretanto, não houve diferença estatística $(p=0,29)$.

Quanto à localização do tumor, notamos maior incidência no reto e no sigmóide. Embora entre os mais jovens tenhamos observado mais tumores no lado direito do cólon. A avaliação estatística não mostrou diferença. $(p=0,65)$ (Tabela 2 e Gráfico 2)

O estádio do tumor em ambos os grupos revelou poucas lesões precoces e a comparação entre os estádios não mostrou diferença estatística. $(p=0,26)$ (Tabela 3). Observamos o mesmo, quando compara-

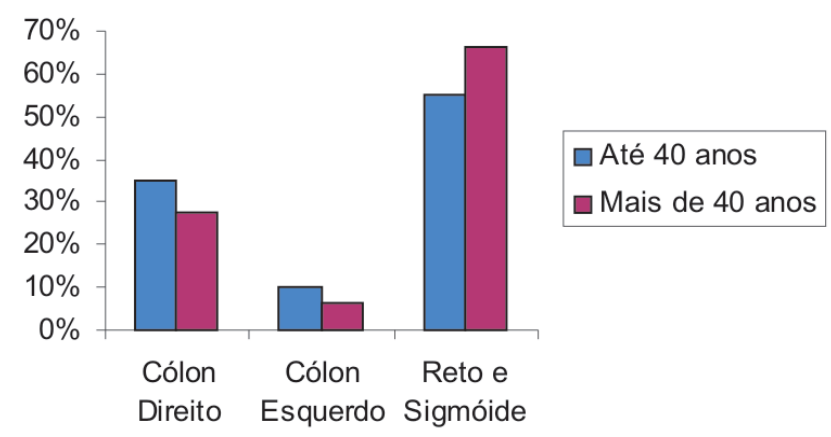

Gráfico 2 - Locais x Idade. mos os dois grupos quanto à invasão tumoral na parede da víscera $(\mathrm{T}) .(p=1,00)$

A comparação entre os grupos em relação à diferenciação tumoral mostrou que os tumores entre os mais jovens foram mais indiferenciados $(p=0,03)$, embora não tenhamos observado mais lesões com componente mucinoso ou com células em anel de sinete em qualquer dos grupos. (Tabela 4)

As mortes até o $30^{\circ}$ dia ocorreram em 13 doentes do grupo 2 e em um do grupo 1 , não havendo diferença estatística $(p=0,73)$. Notamos o mesmo, quanto à mortalidade além dos três anos. $(p=0,97)$ (Tabela 5).

As recidivas ocorreram em quatro $(20 \%)$ dos doentes do grupo 1 e em $23(12,5 \%)$ do grupo 2, resultado sem significância estatística $(p=0,55)$.

Tabela 3 - Distribuição de 205 doentes de carcinoma colorretal conforme o grupo etário e o estádio do tumor. 2006.

\begin{tabular}{lrrrrr}
\hline Estádio & $\begin{array}{c}\text { Até } \\
\mathbf{n}^{\mathbf{0}}\end{array}$ & $\begin{array}{r}\mathbf{4 0} \\
\mathbf{\%}\end{array}$ & $\begin{array}{r}\text { Mais de } \\
\mathbf{n}^{\mathbf{0}}\end{array}$ & $\begin{array}{c}\text { 40 anos } \\
\mathbf{\%}\end{array}$ & $\mathbf{p}$ \\
\hline I & 1 & $6,3 \%$ & 17 & $10,9 \%$ & 0,88 \\
II & 8 & $50,0 \%$ & 47 & $30,1 \%$ & 0,18 \\
III & 5 & $31,3 \%$ & 37 & $23,7 \%$ & 0,72 \\
IV & 2 & $12,5 \%$ & 55 & $35,3 \%$ & 0,12 \\
Total & 16 & $100,0 \%$ & 156 & $100,0 \%$ & \\
\hline
\end{tabular}

Tabela 2 - Distribuição de 204 doentes de carcinoma colorretal conforme o grupo etário e o sítio do tumor. 2006.

\begin{tabular}{lccccc}
\hline Local & \multicolumn{2}{c}{ Até } & $\mathbf{4 0}$ anos & \multicolumn{2}{c}{ Mais de } \\
& $\mathbf{n}^{\mathbf{0}}$ & $\mathbf{4 0}$ & $\mathbf{a n o s}$ & $\mathbf{p}$ \\
\hline Ceco & 3 & $15 \%$ & 19 & $10,4 \%$ & 0,80 \\
Cólon Ascendente & 1 & $5 \%$ & 14 & $7,6 \%$ & 0,98 \\
Ângulo Hepático & 3 & $15 \%$ & 12 & $6,5 \%$ & 0,35 \\
Cólon Transverso & 0 & - & 4 & $2,2 \%$ & - \\
Ângulo Esplênico & 0 & - & 1 & $0,5 \%$ & - \\
Cólon Descendente & 2 & $10 \%$ & 10 & $5,4 \%$ & 0,74 \\
Sigmóide & 3 & $15 \%$ & 33 & $18,0 \%$ & 0,98 \\
Reto & 8 & $40 \%$ & 86 & $46,8 \%$ & 0,73 \\
Sincrônicos & 0 & - & 2 & $1,0 \%$ & - \\
S/ informação & 0 & - & 3 & $1,6 \%$ & - \\
Total & 20 & $100 \%$ & 184 & $100,0 \%$ & \\
\hline
\end{tabular}


Tabela 4 - Distribuição de 205 doentes de carcinoma colorretal conforme o grupo etário e o grau de diferenciação do tumor. 2006.

\begin{tabular}{lrrrrr}
\hline Diferenciação histológica & \multicolumn{2}{c}{ Até $\mathbf{4 0}$ anos } & \multicolumn{2}{c}{ Mais de $\mathbf{4 0}$ anos } & p \\
& $\mathbf{n}^{\mathbf{0}}$ & $\mathbf{\%}$ & $\mathbf{n}^{\mathbf{0}}$ & \multicolumn{1}{c}{$\mathbf{\%}$} & \\
\hline Bem diferenciado & 1 & $5 \%$ & 20 & $10,9 \%$ & 0,66 \\
Moderadamente diferenciado & 11 & $55 \%$ & 119 & $64,7 \%$ & 0,55 \\
Pouco Diferenciado & 2 & $10 \%$ & 8 & $3,3 \%$ & 0,39 \\
Carcinoma Indiferenciado & 0 & - & 4 & $2,2 \%$ & - \\
C/ Células em anel de sinete & 1 & $5 \%$ & 1 & $0,5 \%$ & 0,48 \\
Mucinoso & 4 & $20 \%$ & 15 & $8,1 \%$ & 0,18 \\
Não houve peça & 1 & $5 \%$ & 5 & $2,7 \%$ & - \\
A.P. não encontrado & 0 & - & 12 & $6,6 \%$ & - \\
Total & 20 & & 184 & $100,0 \%$ & \\
\hline
\end{tabular}

Tabela 5 - Distribuição de 205 doentes de carcinoma colorretal conforme o grupo etário e a época de morte pós-operatoria. 2006.

\begin{tabular}{|c|c|c|c|c|c|}
\hline \multirow[t]{2}{*}{ Estádio } & \multicolumn{2}{|c|}{ Até 40 anos } & \multicolumn{2}{|c|}{ Mais de 40 anos } & \multirow[t]{2}{*}{$\mathbf{p}$} \\
\hline & $\mathbf{n}^{\mathbf{o}}$ & $\%$ & $\mathbf{n}^{\mathbf{0}}$ & $\%$ & \\
\hline$<30$ dias & 1 & $20 \%$ & 14 & $24,1 \%$ & 0,73 \\
\hline 30 dias a 3 anos & s 3 & $60 \%$ & 39 & $67,3 \%$ & 0,87 \\
\hline$>3$ anos & 1 & $20 \%$ & 5 & $8,6 \%$ & 0,97 \\
\hline Total & 5 & $100 \%$ & 58 & $100,0 \%$ & \\
\hline
\end{tabular}

As metástases no grupo 1 foram predominantemente abdominais enquanto que no grupo 2 , as hepáticas e as pulmonares foram as mais comuns $(p<0,01)$ (Tabela 6).

\section{DISCUSSÃO}

Geralmente essa neoplasia aparece após a sexta década da vida. Entretanto, 2 a $8 \%$ dos tumores colorretais surgem em doentes com menos que 40 anos de idade..$^{24,25}$ Nossa casuística mostrou incidência de $9,75 \%$ de doentes nessa faixa mais jovem.

A detecção do CCR em jovens não deve ser diferente da forma usada nos doentes mais idosos, mas necessita de elevado índice de suspeita. ${ }^{26}$ Todavia, esse tipo de carcinoma vem sendo diagnosticado em estádios mais avançados entre os mais jovens. ${ }^{27-29}$ Entre nossos doentes, observamos que os mais jovens demoram mais para procurar auxílio médico. Entretanto, devemos considerar que esse diagnóstico geralmente é considerado como exceção nesse grupo.

Tabela 6 - Distribuição de 205 doentes de carcinoma colorretal conforme o grupo etário e o local de aparecimento da metástase mais distante. 2006.

\begin{tabular}{|c|c|c|c|c|c|c|c|c|}
\hline \multirow{2}{*}{ Local } & \multirow[b]{2}{*}{$n^{\mathbf{o}}$} & \multicolumn{2}{|c|}{ até 40 anos } & \multicolumn{3}{|c|}{ Mais de 40 anos } & \multirow{2}{*}{ p Meta } & \multirow{2}{*}{ p Tota } \\
\hline & & $\%$ Meta & $\%$ Total & $\mathbf{n}^{\mathbf{o}}$ & $\%$ Meta & $\%$ Total & & \\
\hline Abdominais & 3 & $75 \%$ & $15 \%$ & 5 & $7,8 \%$ & $2,7 \%$ & $<0,01$ & 0,04 \\
\hline Fígado & 1 & $25 \%$ & $5 \%$ & 34 & $53,1 \%$ & $18,5 \%$ & 0,56 & 0,23 \\
\hline Pulmão & 0 & - & - & 18 & $28,1 \%$ & $9,8 \%$ & - & - \\
\hline Cérebro & 0 & - & - & 3 & $4,7 \%$ & $1,6 \%$ & - & - \\
\hline Ovário & 0 & - & - & 2 & $3,1 \%$ & $1,1 \%$ & - & - \\
\hline Outros & 0 & - & - & 2 & $3,1 \%$ & $1,1 \%$ & - & - \\
\hline Total & 4 & $100 \%$ & $20 \%$ & 64 & $100,0 \%$ & $34,7 \%$ & & \\
\hline
\end{tabular}


A história familiar do CCR esteve presente entre $5 \%$ e $14 \%$ de todos os doentes ${ }^{30,3,1}$ e em $15 \%$ daqueles com menos que 40 anos, ${ }^{30}$ quando aplicados os critérios de Amsterdam. Tivemos incidência de relatos de neoplasia familial em $31,5 \%$ do grupo mais idoso e em $50 \%$ dos mais jovens. Aqui consideramos as neoplasias malignas em qualquer órgão e em qualquer parente das linhas horizontal e vertical. Sugerimos que a obtenção desses índices ocorreu devido às famílias em nosso país serem geralmente numerosas e a chance de seus membros apresentarem tumores malignos ser maior.

Análise da literatura, quanto à localização dos tumores comparando doentes acima e abaixo de 40 anos, não mostrou diferença, ${ }^{25,32-34}$ corroborando com nossa experiência, embora haja relato de que a localização retal é mais comum entre os mais idosos. ${ }^{27}$

Revisão da literatura mostrou que o CCR na população mais jovem é mais agressivo, é diagnosticado nos estádios mais avançados ${ }^{27-29} \mathrm{e}$ tem achados histopatológicos mais indiferenciados. ${ }^{27,28}$

Consideramos como tumores mais indiferenciados aqueles com componente mucinoso, os com células em anel de sinete e os adenocarcinomas pouco diferenciados. O componente mucinoso e as células em anel de sinete são mais comuns no grupo mais jovem. ${ }^{34,35} \mathrm{O}$ componente mucinoso é um dos fatores que influencia a sobrevivência dos doentes com CCR. O adenocarcinoma mucinoso é caracterizado pela presença de mucina extracelular que corresponda a mais que $50 \%$ do volume do tumor. Predomina em homens, tem localização preferencial no cólon direito, apresenta-se em estádios mais avançados, mais metástases peritoneais, embora com menos metástases hepáticas e menor taxa de ressecção curativa, embora a sobrevivência em 5 anos seja pior, ${ }^{34,36-38}$ como em todos os tumores mais indiferenciados.

Tumores pouco diferenciados proliferam e produzem metástases mais rápido que os bem diferenciados. São maiores que os bem ou moderadamente diferenciados. A incidência de metástases linfonodais também é maior. Exceto pelo elevado índice de tumores mais indiferenciados em doentes jovens, não houve di- ferenças na comparação com os mais idosos. As taxas de mortalidade não revelaram prognóstico menos favorável para os mais jovens, a exemplo do citado por outros. $^{32}$

A literatura mostrou que os tumores estádio III, na classificação TNM, foram mais comuns nos doentes mais jovens e as lesões estádio II foram mais frequentes nos acima dos 45 anos. ${ }^{29} \mathrm{Em}$ nossos casos, observamos que o estádio II foi mais comum no grupo dos mais jovens e o IV entre os mais velhos, entretanto sem diferença estatística. Isso ocorreu, talvez, porque os doentes demoraram para procurar a consulta, ou tiveram o diagnóstico retardado, permitindo que o tumor evoluísse. Mas, em ambos, a comparação das taxas de sobrevivência foram maiores para os doentes com menos que 45 anos de idade, tanto para todo o grupo, como na avaliação de cada estádio isoladamente. ${ }^{29}$ Esse estudo confirmou que a idade não foi fator de pior prognóstico para os doentes jovens. O estádio da doença no momento do diagnóstico foi o único fator independente para melhor sobrevivência nos mais jovens, ${ }^{29}$ ressaltando a importância de rastreamento familiar agressivo e diagnóstico precoce nos mais jovens.

A análise dos trabalhos consultados, comparando doentes com idade superior e inferior a 40 anos, mostrou que o sexo feminino, os estádios avançados ao diagnóstico e a localização retal foram mais comuns entre os mais velhos; e os tumores no lado direito, os mais indiferenciados e o diagnóstico inicial com complicação, os mais incidentes entre os mais jovens, ${ }^{29}$ embora haja controvérsias quanto ao sítio do tumor. ${ }^{25,33,34}$

Nosso estudo confirmou que a idade não foi fator de pior prognóstico para o CCR em doentes jovens, como comentado por outros,${ }^{26}$ uma vez que as taxas de mortalidade e metástases foram semelhantes.

Os resultados obtidos nas condições de execução do presente estudo, em que comparamos doentes portadores de adenocarcinoma colorretal com idades acima e abaixo de 40 anos, permitiram concluir que os tumores foram mais indiferenciados entre os mais jovens embora a evolução pós-tratamento tenha sido semelhante. 
Rev bras Coloproct Julho/Setembro, 2009
Luis Roberto Manzione Nadal e Cols.

\begin{abstract}
OBJECTIVE: High incidence of colorectal carcinoma turns it into a public health problem in our country. A few articles, as well as some doubts about patients age and disease evolution, made us study these features to know about tumor cells differentiation and tumor staging in the post-operative follow-up, comparing patients younger and older than 40 years old. METHOD: Comparison of $\mathbf{2 0 5}$ colorectal carcinoma patients younger and older than $\mathbf{4 0}$ years according to symptoms duration, familial history, tumor site, tumor stage, tumor cells differentiation, operative death, metastases site and mortality up to the third year. RESULTS: There were 20 in the younger group and 185 in the older group. There was no difference according to gender, symptoms duration, familial history, tumor site, tumor stage, cancer recurrence, operative mortality and surviving up to the third post-operative year. Tumors were less differentiated and abdominal metastases were more frequent in the younger group. In the older group, hepatic and pulmonary metastases were more common. CONCLUSION: Results obtained in execution conditions of this study, comparing colorectal cancer patients with ages under and over 40 years old, allowed us to conclude that tumors were less differentiated among the younger group despite post-operative evolution having been similar.
\end{abstract}

Key words: Adenocarcinoma, Colorectal Neoplasias, Neoplasia Staging, Cell Differentiation.

\section{REFERÊNCIAS}

01. Casciato DA, Lowitz BB. Manual of Clinical Oncology. $4^{\text {th }}$ Edition. Lippincott Williams \& Wilkins Inc 2001:182

02. Liang H, Wang XN, Wang BG, Pan Y, Liu N, Wang DC, et al. Prognostic factors of young patients with colon cancer after surgery. World J Gastroenterol 2006;12:1458-1462.

03. Fundação Oncocentro de São Paulo. Mortalidade por Câncer no Estado de São Paulo. Imprensa Oficial, São Paulo, 2005, $202 \mathrm{p}$.

04. Ministério da Saúde. Instituto Nacional do Câncer (INCA). Atlas mostra panorama da mortalidade por câncer no Brasil. Disponível em http://www.inca.gov.br/releases/2002/ press_atlas.html.

05. Parramore JB, Wei JP, Yeh KA. Colorectal cancer in patients under forty: presentation and outcome. Am Surg 1998;64:5637.

06. Monteiro EP, Salem JB, Taglietti EM, Albuquerque IC, Formiga GJS. Neoplasia colorretal até 40 anos - Experiência em cinco anos. Rev bras Coloproct 2006;26(2):156-161.

07. Drumond CA, Ferro RAF, Nogueira AMF, Profeta da Luz MM, Conceição SA, Silva RG, et al. Câncer colorretal em pacientes com idade inferior a 30 anos. Rev bras Coloproct, 2003;23(3):147-154

08. Simstein NL, Kovalcik PJ, Cross GH. Colorectal carcinoma in patients less than 40 years old. Dis Colon Rectum 1978; 21:169-171.

09. Okuno M, Ikehara T, Nagayama M. Colorectal carcinoma in young adults. Am J Surg 1987; 154:264-268.

10. Howard EW, Carvalho C, Hovey LM, Nelson TS. Colon and rectal cancer in the young adult. Am Surg 1975; 41:260-264.

11. Safford KL, Spebar MJ, Rosenthal D. Review of colorectal cancer in patients under age 40 years. Am J Surg 1981;142:767769.

12. Ezzo JA, Sullivan JF, Mack RE. Carcinoma of the colon under the age of 40. Ann Intern Med 1958; 49:321-325.
13. Hall A, Coffey RJ. Cancer of the large bowel in the young adult. Am J Surg.1961; 102: 66-72.

14. Rosato FE, Frazier TG, Copeland EM, Miller LD. Carcinoma of the colon in young people. Surg Gynecol Obstet 1969; 129: 29-32.

15. Ohman ULF. Colorectal carcinoma in patients less than 40 years of age. Dis Colon Rectum 1982; 25:209-214.

16. Mitry E, Bouvier AM, Esteve J, Faivre J. Improvement in colorectal cancer survival: a population-based study. Eur J Cancer. 2005;41:2297-303.

17. Ciccolallo L, Capocaccia R, Coleman MP, Berrino F, Coebergh JW, Damhuis RA, et al. Survival differences between European and US patients with colorectal cancer: role of stage at diagnosis and surgery. Gut. 2005;54:268-73.

18. Safford KL, Spebar M, Rosenthol D. Review of colorectal cancer in patients under age 40 years. Am J Surg 1981;142:767769.

19. Moore PA, Dilawari RA, Fidler WJ. Adenocarcinoma of the colon and rectum in patients less than 40 years of age. Am Surg 1984;50:10-14.

20. Liang H, Wang XN, Wang BG, Pan Y, Liu N, Wang DC, et al.. Prognostic factors of young patients with colon cancer after surgery. World J Gastroenterol. 2006;12:1458-62.

21. Sanfelippo PM, Beahrs OH. Carcinoma of the colon in patients under forty years of age. Surg Gynecol Obstet 1974;138:169170.

22. Goi T, Hirono Y, Katayama K, Yamaguchi A. Microsatellite instability and survival rate in the solid or nonsolid types of poorly differentiated colorectal adenocarcinoma. Int Surg. 2004;89:100-6.

23. Ponz de Leon M, Di Gregorio C. Pathology of colorectal cancer. Dig Liver Dis. 2001; 33:372-88.

24. Miccini M, Mazzoni G, Cassini D, Bettelli E, Colace L, De Angelis M, et al. Colorectal carcinoma in the young. Prognostic factors. G Chir. 2005;26:365-70.

25. Chiang JM, Chen MC, Changchien CR, Chen JS, Tang R, Wang JY, et al. Favorable influence of age on tumor 
characteristics of sporadic colorectal adenocarcinoma: patients 30 years of age or younger may be a distinct patient group. Dis Colon Rectum. 2003;46:904-10.

26. Chung YF, Eu KW, Machin D, Ho JM, Nyam DC, Leong AF, et al. Young age is not a poor prognostic marker in colorectal cancer. Br J Surg. 1998; 85:1255-9.

27. Al-Jaberi TM, Yaghan RJ, El-Heis HA. Colorectal cancer in young patients under 40 years of age. Comparison with old patients in a well defined Jordanian population. Saudi Med J. 2003;24:871-4

28. O'Connell JB, Maggard MA, Livingston EH, Yo CK. Colorectal cancer in the young. Am J Surg. 2004;187:343-8.

29. Mitry E, Benhamiche AM, Jouve JL, Clinard F, Finn-Faivre C, Faivre J. Colorectal adenocarcinoma in patients under 45 years of age: comparison with older patients in a well-defined French population. Dis Colon Rectum. 2001;44:380-7

30. Makela J, Kiviniemi H, Laitinen S. Prognostic factors after surgery in patients younger than 50 years old with colorectal adenocarcinoma. Hepatogastroenterology 2002;49:971-5.

31. Olschwang S, Bonaiti-Pellie C, Feingold J, Frebourg T, Grandjouan S, Lasset C, et al. Identification and management of HNPCC syndrome (hereditary non polyposis colon cancer), hereditary predisposition to colorectal and endometrial adenocarcinomas. Pathol Biol (Paris). 2006;54:215-229

32. Frizis H, Papadopoulos A, Akritidis G, Frizis HR, Hatzitheoharis G. Are there any differences in colorectal cancer between young and elderly patients? Tech Coloproctol. 2004;8 Suppl 1:s147-8
33. Paraf F, Jothy S. Colorectal cancer before the age of 40: a casecontrol study. Dis Colon Rectum. 2000;43:1222-6.

34. Tung SY, Wu CS, Chen PC. Primary signet ring cell carcinoma of colorectum: an age- and sex-matched controlled study. Am J Gastroenterol. 1996;91:2195-9.

35. de Silva MV, Fernando MS, Fernando D. Comparison of some clinical and histological features of colorectal carcinoma occurring in patients below and above 40 years. Ceylon Med J. 2000;45:166-8.

36. Papadopoulos VN, Michalopoulos A, Netta S, Basdanis G, Paramythiotis D, Zatagias A, et al. Prognostic significance of mucinous component in colorectal carcinoma. Tech Coloproctol. 2004;8 Suppl 1:s123-5.

37. Nozoe T, Anai H, Nasu S, Sugimachi K. Clinicopathological characteristics of mucinous carcinoma of the colon and rectum. J Surg Oncol. 2000;75:103-7.

38. Psathakis D, Schiedeck TH, Krug F, Oevermann E, Kujath P, Bruch HP. Ordinary colorectal adenocarcinoma vs. primary colorectal signet-ring cell carcinoma: study matched for age, gender, grade, and stage. Dis Colon Rectum.1999;42:1618-25.

Endereço para correspondência:

LUIZ ROBERTO MANZIONE NADAL

Rua Mateus Grou, 130

CEP 05415-040

São Paulo - SP 